\title{
Utilização da lavagem gástrica e do carvão ativado como medidas de intervenção terapêutica na intoxicação exógena
}

\author{
Use of gastric lavage and activated charcoal as measures of therapeutic intervention in \\ exogen poisoning
}

\section{Uso de lavado gástrico y carbón activado como medidas de intervención terapéutica en intoxicación por exógenos}

Anne Caroline Pereira Bezerra ${ }^{1 *}$, Cristiana Barbosa da Silva Gomes ${ }^{1}$, Luana de Macêdo², Maria d' Ávilla Oliveira Dantas Diniz ${ }^{3}$, Rayelle Tássia Azevedo de Caldas², Tayse Gabrielly Leal da Silva ${ }^{1}$, Tcharlys Lopes de Oliveira ${ }^{1}$, Saulo Rios Mariz ${ }^{4}$.

\section{RESUMO}

Objetivo: Descrever um levantamento bibliográfico acerca da utilização da Lavagem Gástrica (LG) e do Carvão Ativado (CA) como medida de descontaminação gastrintestinal em pacientes com intoxicação exógena. Métodos: Trata-se de uma revisão integrativa a partir da consulta dos trabalhos indexados nas plataformas de pesquisas Scientific Electronic Library Online, Biblioteca Virtual em Saúde e Coordenação de Aperfeiçoamento de Pessoal de Nível Superior, com os Descritores em Ciências da Saúde, "lavagem gástrica" ON "carvão ativado" AND "intoxicação exógena". Critérios de inclusão: artigos publicados nos últimos cinco anos, com texto completo ou resumos disponíveis gratuitamente; nos idiomas inglês, espanhol ou português; e que se relacionassem com o objetivo da pesquisa. Resultados: Divididos em estudos que referenciaram um princípio ativo intoxicante e outros que referenciaram vários, a maioria dos autores não esclarece os benefícios e malefícios da terapêutica, independente do tempo de ingestão e administração ou não do CA e LG, todavia, observa-se que o uso de CA é demonstrado como método preferível de descontaminação. Considerações Finais: O CA e a LG são os métodos mais utilizados para descontaminação gastrintestinal em pacientes com intoxicação exógena, mesmo havendo divergência entre o tempo da ingesta do agente tóxico e a utilização destes procedimentos.

Palavras-chave: Lavagem gástrica, Intoxicação, Carvão ativado.

\section{ABSTRACT}

Objective: To describe a bibliographic survey about the use of gastric lavage (LG) and activated carbon (CA) as a measure of gastrointestinal decontamination in patients with exogenous intoxication. Methods: This is an integrative review based on the consultation of works indexed in the research platforms Scientific Electronic Library Online, Virtual Health Library and Coordination for the Improvement of Higher Education Personnel, with the Health Sciences Descriptors, "gastric lavage "ON" activated carbon "AND" exogenous intoxication ". Inclusion criteria: articles published in the last five years, with full text or abstracts available for free; in English, Spanish or Portuguese; and related to the research objective. Results: Divided into studies that referenced an intoxicating active principle and others that referred to several, most authors do not clarify the benefits and harms of the therapy, regardless of the time of ingestion and administration or not of CA and LG, however, it's observed that the use of CA is demonstrated as a preferable method of decontamination. Final Considerations: CA and LG are the most used methods for gastrointestinal decontamination in patients with exogenous intoxication, even though there is a divergence between the time of ingestion of the toxic agent and the use of these procedures.

Keywords: Gastric lavage, Intoxication, Activated carbon.

\footnotetext{
${ }^{1}$ Universidade Federal de Campina Grande (UFCG), Campina Grande - PB.

*E-mail: annecarolinepb@outlook.com

2 Universidade Estadual da Paraíba (UEPB), Campina Grande - PB.

${ }^{3}$ Centro Universitário de Patos (UNIFIP), Patos - PB.

${ }^{4}$ Université de Paris, Paris - FR.
} 


\section{RESUMEN}

Objetivo: Describir una encuesta bibliográfica sobre el uso de Lavado Gástrico (LG) y Carbón Activado (CA) como medida de descontaminación en pacientes que padecen intoxicación exógena. Métodos: Se trata de una revisión integradora basada en consulta de trabajos indexados en las plataformas de investigación Biblioteca Electrónica Científica en Línea, Biblioteca Virtual en Salud y Coordinación para Perfeccionamiento del Personal de Educación Superior, con los Descriptores: "lavado gástrico", "carbón activado" y "intoxicación exógena". Criterios de inclusión: artículos publicados en los últimos cinco años, con texto completo, esúmenes disponibles de forma gratuita; en inglés, español o portugués; y relacionados con el objetivo de la investigación. Resultados: Divididos en estudios que hicieron referencia a un principio activo intoxicante y otros que mencionaron varios, la mayoría de autores no aclaran los beneficios y perjuicios de la terapia, independientemente del momento de ingestión y administración o no de CA y LG, sin embargo, se observa que el uso de CA se demuestra como un método preferible de descontaminación. Consideraciones Finales: CA y LG son los métodos más utilizados para la descontaminación en pacientes que sufren intoxicación exógena, aunque existe una divergencia entre el momento de ingestión del agente tóxico y el uso de estos procedimientos.

Palabras clave: Lavado gástrico, Intoxicación, Carbón activado.

\section{INTRODUÇÃO}

De acordo com dados do Sistema de Informação de Agravos de Notificação (SINAN) e sua última atualização, realizada no dia 13/05/2020, foram notificados 46.082 registros de Intoxicação Exógena (IE) no Brasil neste ano. Devido ao crescente número de casos, esse tema resulta em um importante problema de saúde pública, tendo em vista os impactos negativos gerados ao indivíduo e a sociedade (DATASUS, 2020).

Ademais, dentre as principais vias de exposição nos casos de IE está a via oral, sendo utilizada como meio para a ingesta intencional ou acidental de agentes tóxicos, sejam esses, medicamentos, alimentos, plantas tóxicas, agrotóxicos, drogas de abuso, entre outros, os quais requerem intervenções terapêuticas emergenciais, como forma de melhorar a clínica do paciente e garantir sua sobrevida (MINTEGI S, et al., 2017).

Nesse cenário adentra as medidas de descontaminação gastrintestinal, como forma de promover a evacuação e/ou diminuir a absorção do agente tóxico pelo trato gastrintestinal. Temos como exemplo de medidas utilizadas nos serviços de emergência para esse objetivo o Xarope de Ipeca, os Catárticos, a Irrigação Intestinal, a Lavagem gástrica (LG) e administração do Carvão Ativado (CA), sendo esses dois últimos os mais comuns (MINTEGI S, et al., 2017).

A LG é um procedimento terapêutico realizado para diminuir a absorção de agentes tóxicos, por ingestão de alimentos, medicamentos, domissanitários, entre outros, bem como remover secreções irritantes à mucosa gástrica. Consiste na irrigação e aspiração do conteúdo estomacal por meio de uma sondagem gástrica e é indicada, no geral, para tratamento de intoxicações exógenas e em casos de preparo para exames e algumas cirurgias (MANUAL, 2016).

Além disso, é importante enfatizar que o emprego da LG pode acarretar alguns riscos para o paciente. Citam-se na literatura a ocorrência de laringoespasmo, aspiração de conteúdo gástrico, trauma de esófago ou de vias aéreas, arritmias, translocação de conteúdo gástrico ao intestino delgado, distúrbios hidroeletrolíticos, entre outros problemas ocasionados pelo uso da LG. São relatados ainda, casos em que a introdução de grande quantidade de líquido no estômago pode acarretar distensão gástrica com consequente abertura do piloro e extravasamento de conteúdo para o intestino delgado, fazendo com que, ao invés de reduzir a fração de substância tóxica absorvida pela mucosa do estomago, o procedimento aumente a absorção da droga e, consequentemente, piore o quadro clinico do paciente (LEIDENZ FAB, et., al, 2012).

O CA também denominado de carvão vegetal, carvão animal ou carvão mineral, é um medicamento disponível na lista de Assistência Farmacêutica do Sistema Único de Saúde (SUS) na forma de apresentação em pó ou em comprimido, ambos para uso oral (CETESB, 2018). 
Tem como princípio ativo o carvão vegetal ativado e como principal propriedade unir substâncias à sua superfície, mediante a propriedade de adsorção, o que lhe permite fixar toxinas bacterianas, substâncias químicas irritantes e gases, atuando também como protetor das mucosas (BRASIL, 2013).

Assim como a LG, o CA também pode desencadear complicações para o organismo, tais como, episódios de vômitos, não devendo ser utilizado em pacientes que tenham ingerido substâncias corrosivas, cáusticas ou hidrocarbonetos. Em relação às interações medicamentosas, há registros da ocorrência de hipocalemia, hipernatremia, hipermagnesemia e acidose metabólica, quando o carvão vegetal é administrado com catárticos.

Além disso, a ingestão associada de CA com narcóticos ou compostos anticolinérgicos pode acarretar diminuição do peristaltismo. As reações adversas relacionadas a esse medicamento são principalmente: descoloração das fezes, vômito, como já exposto anteriormente, e o aparecimento de constipação intestinal, sobretudo, quando é utilizado em altas doses (TRENTINI AMM, 2004).

Posto isto, e sabendo que a utilização da LG e/ou CA não é indicada para todos os casos de intoxicação, em que a via de exposição tenha sido a oral, além de se levar em consideração os benefícios e os riscos desses procedimentos, a indicação dos mesmos irá decorrer da presença de alguns fatores determinantes, tais como o tempo decorrido entre a exposição e o atendimento, clínica apresentada, e, principalmente, o tipo de tóxico envolvido.

Essas especificidades acabam gerando algumas divergências do uso dessas intervenções na prática clínica, como por exemplo, uma pesquisa afirma que a utilização do esvaziamento gástrico pode ser realizada em todos os casos de ingestão de tóxicos, desde que em tempo útil, ou seja, antes da absorção digestiva, fazendo com que o tempo médio, em geral, seja entre 2 a 4 horas após a exposição (SILVA P, 2013).

Já segundo os autores Leidenz FAB, et al. (2012), a LG é indicada nos casos de ingestão de substâncias com elevada toxicidade sistêmica e repercussões potencialmente graves; substâncias não adsorvidas pelo carvão ativado; até 60 minutos da ingestão ou em casos específicos de retardo de esvaziamento gástrico somado à alta toxicidade da substância ingerida.

Diante disso, o presente estudo tem como objetivo descrever um levantamento bibliográfico acerca da utilização da Lavagem Gástrica (LG) e do Carvão Ativado (CA) como medida de descontaminação gastrintestinal em pacientes vítimas de intoxicação exógena.

\section{MÉTODOS}

O presente estudo trata-se de uma pesquisa bibliográfica do tipo revisão integrativa. Para a elaboração da pesquisa, foram seguidas as etapas da revisão integrativa: identificação do tema e seleção da hipótese ou questão de pesquisa; estabelecimento dos critérios para inclusão e exclusão de estudos; definição das informações a serem extraídas dos estudos selecionados/categorização dos estudos; avaliação dos estudos incluídos na revisão integrativa; apresentação da revisão/síntese do conhecimento (MENDES KDS, et al., 2008).

No que tange ao levantamento bibliográfico, foram consultados os trabalhos indexados nas seguintes plataforma de pesquisas online: Scielo, BVS e Portal CAPES, com os descritores em português e inglês "lavagem gástrica" ON "carvão ativado" AND "intoxicação".

Os critérios de inclusão da pesquisa foram: artigos publicados nos últimos cinco anos (2015-2020); com texto completo ou resumos disponíveis gratuitamente; publicados nos idiomas inglês, espanhol ou português; e que relacionassem com o objetivo da pesquisa. Foram excluídos os estudos duplicados e que não condiziam com a temática abordada.

Após aplicação dos critérios citados anteriormente, restaram 52 artigos para serem analisados de forma detalhada pelos pesquisadores, sendo distribuídos entre os mesmos, ficando 4 revisores cada qual com 10 artigos, e $05^{\circ}$ revisor com 12 artigos. Por fim, 26 artigos compuseram o estudo. 


\section{RESULTADOS E DISCUSSÃO}

Respeitando os critérios de inclusão encontrou-se 145 registros nas bases de dados. Após leitura dos títulos e resumos, foram excluídos os resultados duplicados (13) e, os artigos que não condiziam com a temática abordada (80). Por fim, 52 artigos foram entregues para os revisores, dos quais apenas 26 publicações permaneceram no estudo, por atenderem ao objetivo do presente estudo (Figura 1).

Figura 1 - Fluxograma da seleção dos artigos segundo critérios de inclusão e exclusão.

Scielo (Scientific Electronic Library Online), BVS (Biblioteca Virtual em Saúde) e portal Capes (Coordenação de Aperfeiçoamento de Pessoal de Nível Superior).

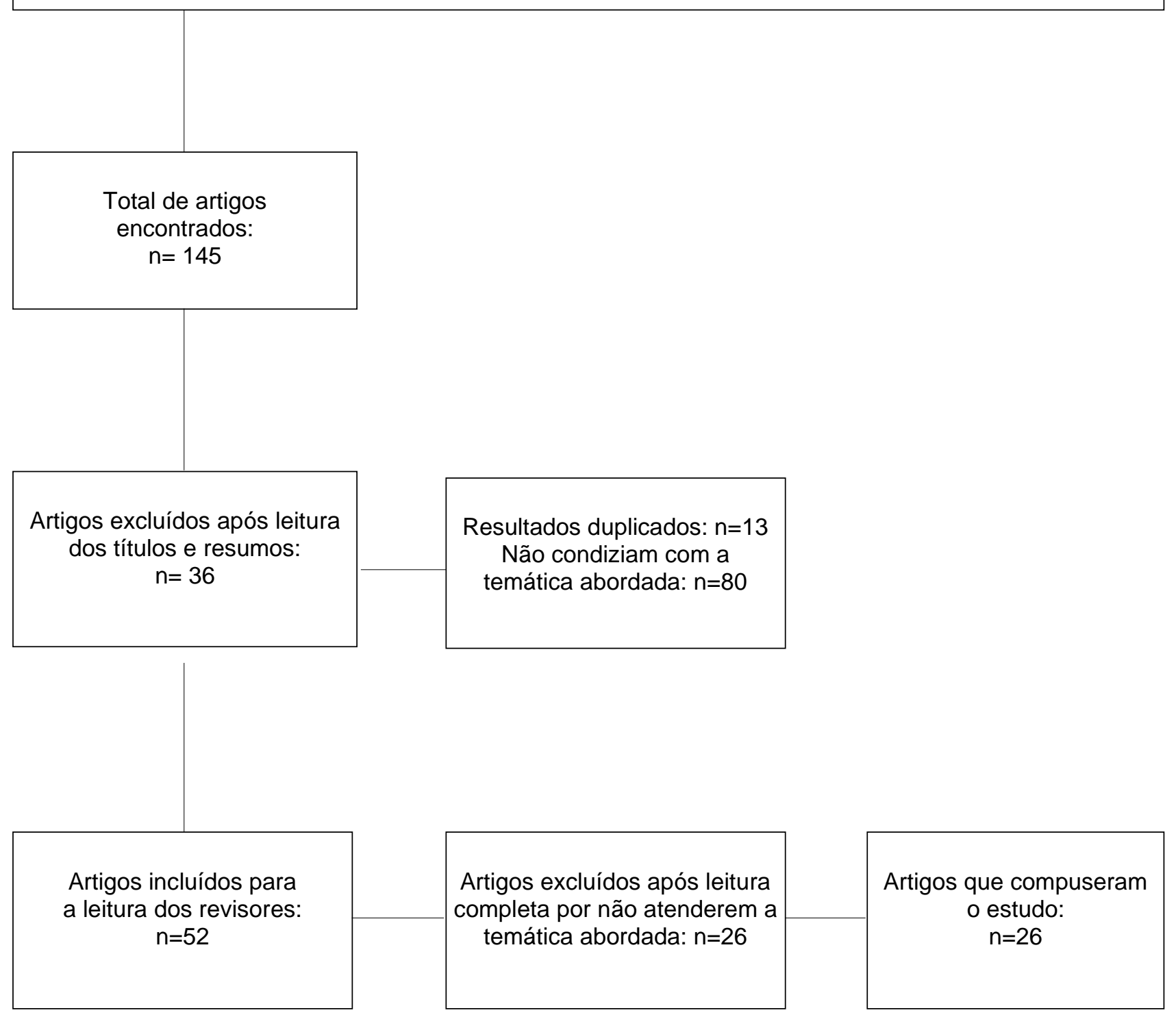

Fonte: BEZERRA ACP, et al., 2020

Utilizou-se um quadro para a coleta dos dados, onde foram colocadas as seguintes informações extraídas da leitura: título, autores, ano de publicação, local, tipo de pesquisa, princípio ativo envolvido, objetivo, medidas de descontaminação (lavagem gástrica e carvão ativado), resultados e discussões. Assim, foi definida a amostra final, em que posteriormente, extraiu-se os dados relevantes para o estudo.

Sendo assim, as informações dos 26 artigos selecionados para compor essa revisão, foram sintetizadas, apresentando os títulos, autores, ano de publicação e tipo de pesquisa de cada estudo (Quadro 1). 


\section{Revista Eletrônica Acervo Saúde / Electronic Journal Collection Health | ISSN 2178-2091}

Quadro 1 - Características das publicações analisadas neste estudo.

\begin{tabular}{|c|c|c|c|}
\hline $\mathbf{N}^{\circ}$ & Titulo & Autores/ Ano & Tipo \\
\hline 1 & $\begin{array}{l}\text { O carvão ativado não reduz a duração da toxicidade da fenitoína em pacientes } \\
\text { hospitalizados. }\end{array}$ & CUMPSTON K, et al., 2016 & Retrospectivo \\
\hline 2 & A farmacocinética da sertralina na sobredosagem e o efeito do carvão ativado. & COOPER JM, et al., 2015 & Farmacocinético \\
\hline 3 & Uma revisão retrospectiva do uso pré-hospitalar de carvão ativado & VILARREAL J, et al., 2015 & Retrospectivo \\
\hline 4 & $\begin{array}{l}\text { Experiência de } 15 \text { anos do Sistema de Controle de Intoxicações da Administração } \\
\text { de Carvão Ativado por Dose Múltipla. }\end{array}$ & CANTRELL FL e SUN C, 2019 & Retrospectivo \\
\hline 5 & $\begin{array}{l}\text { Ingestão tóxica de acetaminofeno e ácido acetilsalicílico em parturiente com } 33 \\
\text { semanas de gestação: Relato de caso }\end{array}$ & REALE SC, et al., 2018 & Relato de caso \\
\hline 6 & $\begin{array}{l}\text { Características clínicas e prognóstico do envenenamento por paraquat na Guiana } \\
\text { Francesa: Uma revisão de } 62 \text { casos. }\end{array}$ & ELENGA N, et al., 2018 & Retrospectivo \\
\hline 7 & Intoxicação aguda por Ferro: Uma entidade frequentemente subvalorizada & CRUZ RM, et al., 2017 & Exploratório/Relato de caso \\
\hline 8 & Convulsão por causa de intoxicacão por múltiplas drogas: Relato de caso & GULEC H, et al., 2016 & Exploratório/ Relato de caso \\
\hline 9 & Quando as sequelas se confundem: Ingestão de cloridrato de Benzidamina & DIAS AL, et al., 2015 & Exploratório/ Relato de caso \\
\hline 10 & $\begin{array}{l}\text { Gerenciamento da intoxicação exógena por Carbonatos e Organofosforados em } \\
\text { uma unidade de emergência }\end{array}$ & LEAO SC, et al., 2015 & Exploratório/ retrospectivo \\
\hline 11 & Sobredosagem maciça de Lidano com análise toxicocinética & WILES DA, et al., 2015 & Exploratório/ Relato de caso \\
\hline 12 & $\begin{array}{l}\text { Análise de Lavagem Gástrica relatada para um sistema estadual de controle de } \\
\text { intoxicações. }\end{array}$ & DONKOR J, et al., 2016 & Retrospectiva \\
\hline 13 & Sobredosagem com Vortioxetina numa Tentativa suicida: Um relato de caso & MAZZA MG, et al., 2018 & Relato de caso \\
\hline 14 & Intoxicação por Cleistanthus Collinus: Relato de caso de intoxicação intencional & BOMPELLI N, et al., 2019 & Estudo de caso / exploratório \\
\hline
\end{tabular}

REAS/EJCH | Vol.12(12) | e4990 | DOI: https://doi.org/10.25248/reas.e4990.2020 Página 5 de 10 


\section{Revista Eletrônica Acervo Saúde / Electronic Journal Collection Health | ISSN 2178-2091}

\begin{tabular}{|c|c|c|c|}
\hline $\mathbf{N}^{\circ}$ & Titulo & Autores/ Ano & Tipo \\
\hline 15 & $\begin{array}{l}\text { Tratamento do envenenamento grave por Fluoroacetamida em pacientes com } \\
\text { síndrome combinada de disfunção de múltiplos órgãos por medicamentos } \\
\text { chineses e ocidentais integrados e baseados em evidências: Relato de caso. }\end{array}$ & WEN W, et al., 2017 & Relato de Caso \\
\hline 16 & $\begin{array}{l}\text { Padrão de envenenamento agudo por organofosforado no Hospital Universitário } \\
\text { de Gondar, Noroeste da Etiópia. }\end{array}$ & ADINEW GM, et al., 2017 & Retrospectivo \\
\hline 17 & Uso De Solução Lipídica Em Intoxicação Por Beta-Bloqueador: Relato De Caso. & MARTINS LD e FILHO AA, 2017 & Relato de caso. \\
\hline 18 & Carvão ativado para overdose aguda: Uma reavaliação & JUURLINK DN, 2015 & Revisão Integrativa \\
\hline 19 & $\begin{array}{c}\text { Identificação e manejo inicial de intoxicações por álcool e outras drogas na sala } \\
\text { de emergência pediátrica }\end{array}$ & PIANCA TG, et al., 2017 & Revisão Integrativa \\
\hline 20 & $\begin{array}{l}\text { Sobredosagem maciça de Paracetamol: Um estudo observacional do efeito do } \\
\text { carvão ativado e aumento da dose de acetilcisteína (ATOM-2) }\end{array}$ & CHIEW AL, et al., 2017 & Observacional \\
\hline 21 & Tratamentos para envenenamento por paracetamol & BUCKLEY NA, et al., 2016 & Estudo de corte \\
\hline 22 & $\begin{array}{c}\text { Um estudo exploratório: Os efeitos terapêuticos da administração pré-misturada } \\
\text { de Carvão-sorbitol ativado em pacientes envenenados com pesticida } \\
\text { Organofosfato }\end{array}$ & MOON J, et al., 2015 & Retrospectivo \\
\hline 23 & $\begin{array}{c}\text { Uso e conhecimento do carvão ativado de dose única: Uma pesquisa com } \\
\text { médicos australianos }\end{array}$ & CORCORAN G, et al., 2016 & Transversal \\
\hline 24 & $\begin{array}{l}\text { Variabilidade internacional em Descontaminação Gastrointestinal com } \\
\text { intoxicações agudas }\end{array}$ & MINTEGI S, et al., 2017 & Transversal \\
\hline 25 & Intoxicação exógena por Carbamato: Relato de caso & MEDEIROS LRFB, et al., 2017 & Relato de caso \\
\hline 26 & Disponibilidade de antídotos no município de Campinas, São Paulo. & FERNANDES LCR, et al., 2017 & Transversal \\
\hline
\end{tabular}

Fonte: BEZERRA ACP et al., 2020.

$$
\text { REAS/EJCH | Vol.12(12) | e4990 | DOI: https://doi.org/10.25248/reas.e4990.2020Ｐágina } 6 \text { de } 10
$$


Verificou-se que, dos 26 artigos analisados, grande parte foram publicados em $2017(n=9)$; percebemos também que estes apresentaram um índice muito relevante de relatos de caso $(n=10)$, esses estudos são muito pertinentes por se tratarem de investigações com desenhos mais elaborados, em que estimulam a interpretação de sinais, sintomas e outras características dos pacientes, como também relatam os procedimentos terapêuticos utilizados, produzindo assim um material vasto para discussões e, assim, contribuir para as decisões terapêuticas no dia-dia dos profissionais de saúde.

Posto isto, na sequência da análise dos artigos selecionados e das evidências científicas encontradas, considerou-se relevante a apresentação de dois eixos temáticos: Estudos que referenciaram apenas um princípio ativo causador da Intoxicação; e Estudos que referenciaram vários princípios ativos causadores das Intoxicações.

\section{Estudos que referenciaram apenas um princípio ativo causador da Intoxicação:}

Nos casos de overdose por Fenitoína, a utilização do CA não reduz a duração da toxidade causada pelo fármaco, ou seja, pode haver pouco ou nenhum efeito clinicamente significativo do uso de única ou múltiplas doses de CA para melhorar a eliminação no paciente intoxicado com esse fármaco (CUMPSTON K, et al., 2016).

Durante um estudo retrospectivo realizado na Austrália, pesquisadores descreveram o manejo terapêutico dos casos de overdose por Sertralina, com ênfase na utilização de dose única do carvão ativado (DUCA), sendo administrado $50 \mathrm{~g}$ dessa substância em $250 \mathrm{ml}$ de água purificada.

O espaço temporal entre o relato de superdosagem e a administração do CA, variou entre 1,5 e $4 \mathrm{~h}$. Os autores concluíram que apesar das diretrizes atuais de tratamento recomendarem o uso de DUCA dentro de $1 \mathrm{~h}$ após a overdose, o uso do mesmo além desse período teve efeito benéfico nos casos de overdose relatados, já que aumentou a depuração da Sertralina (COOPER JM, et al., 2015).

Apenas um caso foi relatado na literatura sobre overdose de Vortioxetina, o trabalho citou a realização de LG com $2 \mathrm{~L}$ de água e o uso do CA de $50 \mathrm{~g}$, não citando o tempo decorrido entre a ingesta e o início desses procedimentos, por fim, o paciente teve melhora clínica e alta hospitalar (MAZZA MG, et al., 2018).

Para as intoxicações por Benzidamina usa-se o tratamento de suporte, com LG e CA até 1h após a ingestão, sendo assim, um estudo relatou o caso de uma criança que ingeriu, acidentalmente, $500 \mathrm{mg}$ de cloridrato de Benzidamina diluída em água $(31,3 \mathrm{mg} / \mathrm{kg})$. Foi feito $L G 2 \mathrm{~h}$ após ingestão, simultaneamente com a administração de CA 1,25g/kg. Após 2 dias de internação o paciente teve alta sem sintomas (DIAS AL, et al., 2015).

A LG não é recomendada para os casos de desintoxicação causadas pelo Ferro, por ser muitas vezes ineficaz para a remoção desses comprimidos do estômago. Essa decisão também deve ser guiada pela clínica apresentada pelo paciente, quantidade de comprimidos que foram ingeridos e sua permanência a nível gástrico, ponderando-os com os possíveis riscos desse procedimento. Ademais, o CA não tem indicação na intoxicação por Ferro, visto que não absorve os íons metálicos (CRUZ RM, et al., 2017).

Já nos casos de intoxicação por Paracetamol não foi citado na literatura a utilização da LG (CHIEW AL, et al., 2017; BUCKLEY NA, et al., 2016; CORCORAN G, et al., 2016), apenas o uso do CA, o qual tem boa indicação terapêutica para esses casos, já que quando feito em até 4 h pós ingestão de superdosagem de paracetamol apresenta taxa significativamente mais baixa desse composto no organismo, em contrapartida aos pacientes intoxicados, que não recebem.

O CA também contribui para taxas menores de hepatotoxicidade (CHIEW AL, et al., 2017). Além disso, um estudo de coorte demonstrou que as pessoas que ingeriram $10 \mathrm{mg}$ de paracetamol e que fizeram uso do CA em menos de $2 \mathrm{~h}$ pós ingesta (uso precoce), reduziram a necessidade da acetilcisteína (BUCKLEY NA, et a., 2016). Outra pesquisa apenas cita uso do CA, mas não especifica dose e nem como foi utilizado (CORCORAN G, CHAN B, CHIEW A, 2016).

Foi encontrado na literatura apenas um caso de envenenamento pela planta Cleistanthus Collinus. No tratamento foi relatado somente a utilização imediata após a internação, do LG e CA, sendo esse último administrado em doses múltiplas após complicações do quadro clínico. 
Não foram relatados a dose e o tempo do CA. Devido às agressivas desordens metabólicas e síndrome do desconforto respiratório agudo ocasionado pela ingesta da referida planta, no caso relatado o paciente foi a óbito no $5^{\circ}$ dia (BOMPELLI N, et al., 2019).

Para os casos de intoxicação por Paraquat, os métodos de descontaminação gástrica mais utilizados foram: a LG e CA. De modo geral, o artigo não informa sobre as condutas que foram utilizadas para o uso desses procedimentos, apenas enfatiza que o CA reduz significativamente a mortalidade nesses casos, recomendando sua utilização dentro da $1^{\underline{a}}$ hora após a ingestão da planta (ELENGA N, et al., 2018).

Para o tratamento de envenenamento grave pelo pesticida Fluoroacetamida, não houve menção ao uso do CA, apenas da LG de forma inespecífica para esses casos, tendo em vista que, erroneamente, durante a admissão o paciente foi diagnosticado com intoxicação por veneno de rato (Brodifacoum), o que dificultou a comprovação da eficácia das decisões terapêuticas subsequentes (WEN W, et al., 2017).

Tratando de inseticidas, o CA e atropina mostram-se bastante eficazes no uso em IE aguda por Carbamatos ou Organofosforatos, sendo recomentado $1 \mathrm{~g} / \mathrm{kg}$ para crianças menores de 1 ano e $50 \mathrm{~g}$ para crianças entre 1 e 12 anos e 25 a 100g para adolescentes e adultos, porém não houve menção a LG nesses casos (LEÃO SC, et al., 2015; MEDEIROS LRFB, et al., 2017). Por outro lado, durante um manejo terapêutico de uma intoxicação causada pela ingesta de Carbamato, foi usado LG com CA por $24 \mathrm{~h}(70 \mathrm{~g} /$ dose $=$ $1 \mathrm{~g} / \mathrm{kg} / \mathrm{dose}$ ) (MEDEIROS LRFB, et al., 2017).

Em contrapartida, um estudo concluiu que os pacientes que receberam CA não obtiveram benefícios, nem malefícios, independentemente do tempo entre a ingestão e a administração. Também não houve menção da LG (MOON J, et al., 2015).

O uso da LG nos casos de intoxicação por organofosforado foi citado em $30 \%$ dos artigos analisados em um estudo retrospectivo, porém não foi citado as especificidades do uso desse procedimento e nem a utilização do CA (ADINEW GM, et al., 2017).

A LG e CA $(30 \mathrm{~g})$ foram utilizados na terapêutica de um paciente vítima de IE por Lindano, de forma imediata, assim como outras medicações para reversão do quadro clínico. O paciente sobreviveu a intoxicação (WILES DA, et al., 2015).

\section{Estudos que referenciaram vários princípios ativos causadores das Intoxicações}

O uso de CA é o método preferido de descontaminação para uma variedade de intoxicantes. $O$ tempo médio decorrido entre a ingestão do intoxicante e a administração de CA foi de 49,8 minutos. O protocolo permite o uso de até $50 \mathrm{~g}$ de CA por ordem permanente ou em consulta no centro de controle de intoxicações ou em um hospital de base. (VILLARREAL J, et al., 2015).

Tratando de intoxicações causadas por álcool e/ou drogas ilícitas, foi encontrado um estudo o qual detalhava vários manejos para desintoxicação desses pacientes, porém não se especificava o uso do CA, apenas não o recomendava para casos de intoxicação por maconha, pois após a ingestão, a maioria dos sintomas demora até três horas, o que limita a eficácia da AC.

O trabalho relata ainda que, em crianças, O CA pode ser necessário para intoxicação acidental de substâncias psicoativas na dose 0,5 a $1 \mathrm{mg} / \mathrm{kg}$, não citando o uso da LG para esses casos (PIANCA TG, et al., 2017).

Para IE causadas pela ingesta de várias drogas, sendo algumas: Carbamazepina, Aspirina, Ácido Valpróico, Etanol, Acetaminofeno, Fenobarbital, Benzodiazepínicos, Ibuprofeno, Teofilina, Dapsona, foi citado o uso da LG com o CA em múltiplas doses na faixa de 15 a $30 \mathrm{~g}$ ou $0,25-1 \mathrm{~g} / \mathrm{kg}$ a cada 2 a 4 horas (CANTRELL FL e SUN C., 2019).

A administração de DUCA (Dose Única de Carvão Ativado) variava de 0,5 a $100 \mathrm{~g}$ em um período de até 6 horas, após ingestão do fármaco. Um estudo concluiu que quanto mais cedo a administração da DUCA, mais a taxa de absorção dos agentes diminuí (JUURLINK DN, 2015). 
A DUCA pode ser considerada para uso em casos envolvendo doses tóxicas, deve ser feita $1 \mathrm{~h}$ após ingestão da substância, com doses múltiplas pode impedir a absorção de alguns medicamentos que persistem no trato gastrointestinal (FERNANDES LCR, et al., 2017).

Um relato de caso de uma gestante que ingeriu Acetaminofeno (APAP) e Ácido acetilsalicílico (ASA), foi publicado, embora não fazendo menção a quantidade. A paciente recebeu doses de $\mathrm{N}$-acetilcisteína (NAC) e duas doses de CA em até 1 hora após o tempo relatado de ingestão de toxinas, não sendo citada a LG.

Por fim, os autores relataram que o gerenciamento imediato desse tipo de intoxicação pode ter melhores resultados maternos e neonatais com o uso de CA e NAC a paciente foi transferida para sala de cirurgia 14 horas após a ingestão de toxinas, tendo boa melhora clínica (REALE SC, et al., 2018).

Outro relato de caso, diz respeito a ingestão em altas doses de Bupropiona, Quetiapina e Levotiroxina. $O$ CA e a LG podem ser aplicados para diminuir a absorção de Bupropiona se o paciente se apresentar para tratamento logo após as primeiras horas de overdose do medicamento. Para o caso apresentado, o uso da LG e CA tiveram grande importância por ter sido feitos nas primeiras horas, diminuindo a absorção das substâncias (GULEC H, et al., 2016).

Outro estudo analisado não detalha o uso da LG, assim como, não cita o CA como método de descontaminação. Concluiu que se faz necessário a educação continuada do profissional médico e demais componentes da equipe de emergência, como forma de auxiliar na seleção dos pacientes no uso ou não da LG, enfatizando interdisciplinaridade durante as tomadas de decisões (DONKOR J, et al., 2016).

Para um caso de IE por Atenolol, Captopril, Losartana e Fluoxetina, a LG foi o método de escolha para diminuição da absorção da droga e o aumento da excreção da fração já absorvida, sendo indicada até no máximo $2 \mathrm{~h}$ após a ingestão. Segundo o texto, o CA é efetivo na adsorção da droga (MARTINS LS e FILHO $A A, 2017)$.

Considera-se que a DUCA é apropriada após a ingestão de um tóxico, exceto aqueles que não são vinculados ao CA, ou quando seu uso não é claramente indicado (pesticidas, potássio, hidrocarbonetos, ácidos, álcalis, álcoois, ferro, inseticidas, lítio e solventes).

Múltiplas doses de CA são potencialmente apropriadas para a ingestão de antimaláricos (quinino), dapsona, carbamazepina, fenobarbital, metilxantinas, fenitoína, digoxina, valproato, nadolol, sotolol, fenilbutazona, tireóide e salicilatos.

A LG sempre foi considerada inadequada, exceto nos casos de ingestão potencialmente letal, quando se sabe que a toxina não se liga à CA. A combinação de CA e LG (e administração de xarope de ipeca) foi considerada inadequada (MINTEGI S, et al., 2017).

Os artigos analisados mostram uma grande divergência quanto à utilização dos procedimentos em questão, principalmente no tocante ao tempo decorrido entre a ingesta do agente tóxico e a utilização desses procedimentos, tendo em vista que um dos principais critérios na definição do emprego de LG e/ou CA, será esse tempo, abrindo precedentes para questionarmos se essas técnicas foram utilizadas de forma correta, já que muitos não referem a dose e o tempo de administração.

\section{CONSIDERAÇÕES FINAIS}

Foi possível constatar que não há um consenso, entre as publicações, analisadas, sobre o uso desses procedimentos de descontaminação e diminuição da absorção de um mesmo toxicante (ou grupo) no tubo digestório. Isso em muito dificulta a agilidade, e consequentemente, a eficiência do tratamento dessas ocorrências. Além do exposto, notou-se uma necessidade maior na documentação das informações que foram determinantes para o uso ou não da LG e CA nos casos relatados, o que acaba por dificultar a padronização dessas condutas terapêuticas, devido à falta de evidencias clinicas mais detalhadas. Desse modo, constata-se a necessidade da continuação do desenvolvimento de pesquisas nesta área, como forma de ampliar o conhecimento e o entendimento dos profissionais de saúde, melhorando assim, a assistência voltada ao paciente vítima de Intoxicação Exógena. 


\section{REFERÊNCIAS}

1. ADINEW GM, et al. Pattern of acute organophosphorus poisoning at university of gondar teaching hospital, Northwest Ethiopia. BMC research notes. 2017; 10(1): 149p.

2. BOMPELLI N, et al. Intoxicação por Cleistanthus Collinus: relato de caso de intoxicação intencional. BMJ Case Reports, Telangana- Ind. 2019; 12(2).

3. BRASIL. Ministério da Saúde. Advocacia Geral da União. Nota técnica n. 275/2013: Carvão vegetal ativado. Brasilia: [s.n]. 2013; 3p.

4. BUCKLEY NA, et al. Treatments for paracetamol poisoning. BMJ. 353. Austrália. 2016. Acesso em maio de 2020. Disponível em: https://www.bmj.com/content/353/bmj.i2579.

5. CANTRELL FL, SUN C. Poison Control System's 15-Year Experience of Multiple Dose-Activated Charcoal Administration. American Journal of Therapeutics. 2018; p.1-2.

6. CHIEW AL, et al. Sobredosagem maciça de paracetamol: uma observação estudo do efeito do carvão ativado e aumento da dose de acetilcisteína (ATOM-2). Clinical Toxicology. 2017; 55; p.1055-1065.

7. COMPANHIA Ambiental do Estado de São Paulo (CETESB). Ficha de Informação de Produto Químico: carvão ativado. São Paulo: CETESB, 2018.

8. COOPER JM, et al. The pharmacokinetics of sertraline in overdose and the effect of activated charcoal. British Journal of Clinical Pharmacology. 2014; 79(2):307-314.

9. CORCORAN G, et al. Use and knowledge of single dose activated charcoal: A survey of Australian doctors. Emergency Medicine Australasia. 2016; 28:578-585.

10. CRUZ RM, et al. Intoxicação Aguda por Ferro: Uma Entidade Frequentemente. Medicina Interna, Lisboa. 2017; 24(4):300-303.

11. CUMPSTON K, et al. Activated Charcoal Does Not Reduce Duration of Phenytoin Toxicity in Hospitalized Patients. American Journal of Therapeutics. 2016; 23(3):771-774.

12. DATASUS, Intoxicação Exógena no Brasil registradas no SINAN NET; Notificações por Circunstância segundo Região de notificação no ano de 2017.2 Disponível em: <http://tabnet.datasus.gov.br/cgi/tabcgi.exe?sinannet/cnv/lntoxbr.def>. Acesso em: 29 maio. 2020.

13. DIAS AL, et al. Quando as saquetas se confundem: ingestão de cloridrato de benzidamina. Nascer e Crescer, Porto. 2015; 24(3):133-135.

14. DONKOR J, et al. Análise de Lavagem Gástrica relatada para um sistema estadual de controle de intoxicações. The Journal of emergency Medicine, 51(4), 394-400.

15. ELENGA N, et al. Clinical features and prognosis of paraquat poisoning in French Guiana. Medicine. 2018; 97(15):17.

16. FERNANDES LCR, et al. Disponibilidade de antídoto no município de Campinas, São Paulo, Brasil. São Paulo Med J. $2017 ; 135(1): 15-22$.

17. GULEC H, et al. Seizure due to multiple drugs intoxication: a case report. Brazilian Journal of Anesthesiology (English Edition). 2016: 66(6):651-653.

18. JUURLINK DN. Activated charcoal for acute overdose: A reappraisal. British Journal of Clinical Pharmacology. 2016; $81(3): 482-487$.

19. LEAO SC, et al. Management of exogenous intoxication by carbamates and organophosphates at an emergency unit. Rev. Assoc. Med. Bras. 2015; 61(5):440-445.

20. LEIDENZ FAB, et al. Lavagem gástrica realmente efetiva? Revista de Medicina Minas Gerais. 2012; 22(8): 37-39.

21. MANUAL. Manual de Procedimentos Operacionais Padrão (Pop) Enfermagem, Secretaria Municipal de Saúde de Campinas - SP. Versão n. 2, 2016.

22. MARTINS LS, FILHO AA. Uso de solução lipídica em intoxicação por beta-bloqueador: relato de caso TT - Use of lipid solution in beta-blocking severe intoxication: case report. Rev. méd. Minas Gerais. 2018; 28:1-5.

23. MAZZA MG, et al. Sobredosagem com vortioxetina numa tentativa suicida - um relato de caso. Medicine, Baltimore. 2018; 97(25).

24. MEDEIROS LRFB, et al. Intoxicação exógena por carbamato: relato de caso. Revista de Medicina da UFC. 2017; 57(2):57-60.

25. MENDES KDS, et al. Integrative review: research method for the incorporation of evidence in health and nursing. Texto Contexto Enferm, 2008;17(4):758-64.

26. MINTEGI S, et al. International variability in gastrointestinal decontamination with acute poisonings. Pediatrics. 2017; $140(2)$.

27. MOON J, et al. Um estudo exploratório; os efeitos terapêuticos da administração pré-misturada de carvão-sorbitol ativado em pacientes envenenados com pesticida organofosfato. Clinical Toxicology. 2015; 53(2).

28. PIANCA TG, et al. Identification and initial management of intoxication by alcohol and other drugs in the pediatric emergency room. Jornal de Pediatria. 2017; 97(1):46-52.

29. REALE SC, et al. Toxic Ingestion of Acetaminophen and Acetylsalicylic Acid in a Parturient at 33 weeks gestation. A \& A Practice. 2018, p.1-3.

30. SILVA P. Farmacologia. Rio de Janeiro: Guanabara Koogan, 2013.

31. TRENTINI AMM. Carvão Ativado: Bula de Medicamento. Herbarium laboratório botânico Ltda. Paraná 2004.

32. VILLARREAL J, et al. A retrospective review of the prehospital use of activated charcoal. The American Journal of Emergency Medicine. 2015; 33(1):56-59.

33. WEN W, et al. Treatment of severe fluoroacetamide poisoning in patient with combined multiple organ dysfunction syndrome by evidence-based integrated Chinese and Western medicines: A case report. Medicine. 2017; 96(27).

34. WILES DA, et al. Sobredosagem maciça de Lindano com Análise Toxicocinética. J. Med. Toxicol. 2015; 11:106-109. 